\title{
JOURNAL OF ENERGY SYSTEMS
}

VOLUME 2, ISSUE 3

DOI: $10.30521 /$ jes. 434224

\section{An adaptive neuro-fuzzy inference system-based approach for daily load curve prediction}

\author{
Abderrezak Laouafi \\ LES Laboratory, Department of Electrical Engineering, University 20 Août 1955-Skikda, Algeria, \\ laouafi_abderrezak@yahoo.fr, orcid.org/0000-0002-5385-7062 \\ Mourad Mordjaoui \\ LRPCSI Laboratory, Department of Electrical Engineering, University 20 Août 1955-Skikda, Algeria, \\ mordjaoui_mourad@yahoo.fr, orcid.org/0000-0002-5195-6016 \\ Taqiy Eddine Boukelia \\ Laboratory of Applied Energetics and Materials, Mechanical Department, Jijel University, Algeria, \\ taqy25000@ hotmail.com, orcid.org/0000-0002-6683-0491
}

Arrived: 20.06.2018 Accepted: 19.09.2018 Published: 30.09.2018

\footnotetext{
Abstract: Forecasting future electricity demand is one of the most important areas in electrical engineering, due to its vital role for secure and profitable operations in power systems. In recent years, the advent of new concepts and technologies such as deregulation of electricity market, smart grids, electric cars and renewable energy integration have introduced great challenges for power system managers and consequently, the field of electric load forecasting becomes more and more important. This paper describes the use of an adaptive neuro-fuzzy inference system approach for daily load curve prediction. The methodology we propose uses univariate modeling to recognize the daily and weekly patterns of the electric load time series as a basis for the forecast. Results from real-world case study based on the electricity demand data in France are presented in order to illustrate the proficiency of the proposed approach. With an average mean absolute percentage error of $2.087 \%$, the effectiveness of the proposed model is clearly revealed.
}

Keywords: Electric load forecasting, Adaptive neuro-fuzzy inference system, Soft computing, Graphical user interface,

Cite this paper as: $\quad$ based approach for daily load curve prediction. Journal of Energy Systems, 2018. 2(3): 115-126, DOI: 10.30521/jes.434224

(C) 2018 Published by peer-reviewed open access scientific journal, JES at DergiPark (www.dergipark.gov.tr/jes) 


\section{INTRODUCTION}

Electric load forecasting is one of the most important areas in electrical engineering, due to its key role for ensuring secure and economic operations in electrical power networks. Electricity providers use load forecasting models to predict the future load and peak demand of their customers in order, to increase or decrease the produced power so as to adjust supply/demand balance at any time in the best conditions of cost and safety. In recent years, the advent of new concepts and technologies such as deregulation of electricity market, smart grids, electric cars and renewable energy integration have introduced great challenges for power system managers and consequently, the topic of electric load forecasting becomes more and more important. According to time horizon, electric load forecasting subject can be broadly divided into four categories: very short-term (from few minutes to an hour), short-term (from an hour to a week), medium-term (from a week to a year) and long-term (for several years).

Forecasting of the annual peak demand expected at several years in the future is indispensable to support the development and the implementation of any power system. This long-term forecast is necessary for power companies to perform grid upgrades and to build new lines and sub-stations. However, there have been only a few works that have addressed the problem of long-term load forecasting [1-4].

Intermediate-term forecasts are used to meet the load requirements for few weeks or few months in advance [5]. This kind of demand forecast has many applications like grid maintenance scheduling, coordination of load dispatching and development of cost-efficient fuel purchasing strategies [6]. Similar to long-term forecasting case, there have been a limited number of works that have addressed the mid-term scale [6-8].

Accurate forecasts for next few minutes to an hour ahead are used by power utilities to ensure real-time load frequency control, real-time security evaluation and economical dispatching functions [9]. In a liberated energy market, utilities tend to maintain the reserves imposed by an independent network operator close to the minimum [10]. Consequently, the slightest error in the prediction could seriously affect production levels, especially for large utilities. Hence, precise forecasts can facilitate the problem of supply/demand balance to a large extent.

Short-term load forecasting (STLF) is an essential element to confirm the overall physical balance between supply and demand for the entire power system. This forecast is usually made to predict daily minimum and maximum electricity demand, total energy and daily load curve. It serves as a basis for unit commitment, hydro-thermal coordination, power exchange, load flow and as an important element for the economic pricing process. Due to the clear interest of short-term electric load forecasting, it is the time horizon selected in this paper.

Several techniques have been proposed for short-term electricity demand forecasting [11]. STLF methods can be typically separated into two main categories: classical methods and artificial intelligence approaches. Classical models include time series analysis [12-13], regression approaches [14] and exponential smoothing techniques [15]. Artificial intelligence models include artificial neural networks [16], fuzzy logic [17] and hybrid approaches [18]. Prediction by conventional techniques such as time series analysis or regression methods still lacks the desired accuracy. In addition, most conventional models are not transferable, which means that the model developed for a site may not be easily modified for use in another utility. On the other hand, the nature of electric load forecasting problem is well suited to the technology of artificial neural networks (ANN) as they can model the complex non-linear relationships through a learning process involving historical data trends. Therefore, several studies have examined the application of ANN to the problem of short-term electric load forecasting [19]. Recently, hybrid neuro-fuzzy models have received a considerable attention from researchers in the field of STLF [20-21]. The neuro-fuzzy modeling approach attempts to combine the merits of neural networks and 
fuzzy logic paradigms into a single capsule. The fuzzy models are based on linguistic rule systems which are simple to understand and transparent to interpretation and analysis, whereas the artificial neuralnetworks models are inspired by human brain and have the ability to learn from experience in order to improve their performance [22]. However, fuzzy systems do not have the learning ability and the fuzzy sets and operators are required to be specified while building the Fuzzy Inference System (FIS). To implement an artificial neural network for a specific task, appropriate network architecture and learning algorithm are required to achieve a successful application of the model. These limitations encouraged the researchers working in different domains to implement combined neuro-fuzzy approaches to attain higher performance by utilizing capabilities of each of neural networks and fuzzy logic techniques [23]. In this context, it is natural to devote the present paper to the application of an Adaptive Neuro-Fuzzy Inference System (ANFIS) approach for short-term electricity demand forecasting.

The paper is organized as follows. Section 2 is devoted to a theoretical description of the adaptive neurofuzzy inference system. Section 3 describes the proposed estimation method. Section 4 provides and explains forecasting results. Finally, Section 5 concludes the paper.

\section{ADAPTIVE NEURO-FUZZY INFERENCE SYSTEM}

An adaptive neuro-fuzzy inference system is a fuzzy inference system implemented in the framework of adaptive networks to facilitate learning and adaptation [24]. The learning algorithm of the adaptive network uses a hybrid rule which combines the gradient method and the least squares estimation to determine premise and consequent parameters of the fuzzy inference system [23]. The role of the hybrid learning algorithm is tuning all the modifiable parameters to achieve a desired input-output mapping [25]. An adaptive network is a feed-forward neural network with supervised learning capability in which each node (neuron) implements a particular function on incoming signals.

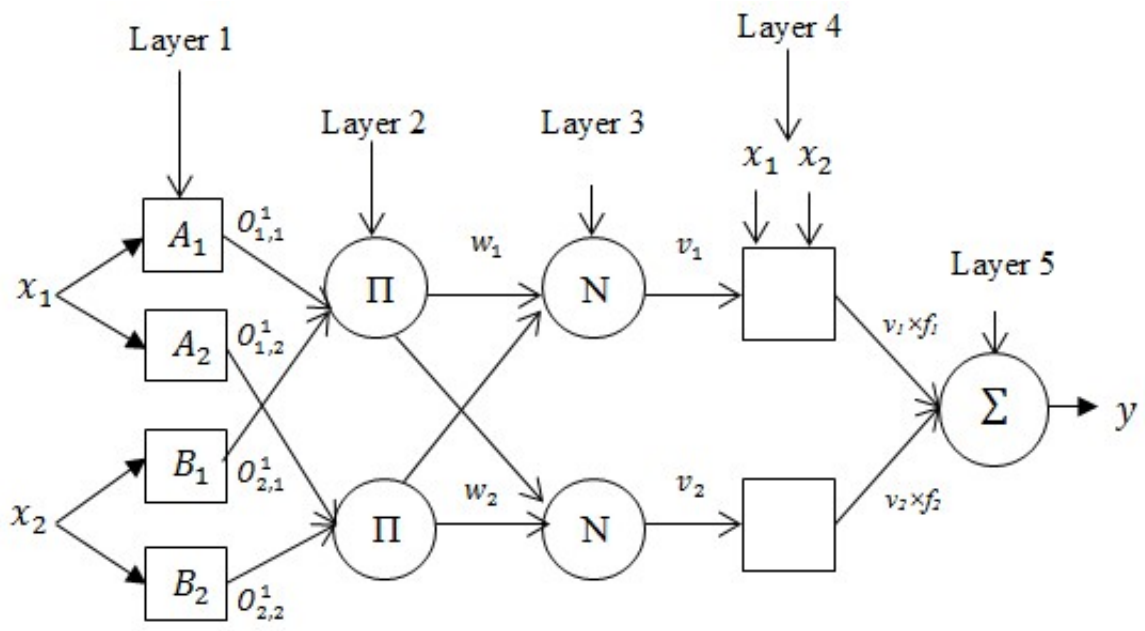

Figure 1. ANFIS architecture

The ANFIS architecture is composed of a premise part and a consequent part, which are connected to each other by a set of Takagi and Sugeno's fuzzy if-then rules in a network form. As shown in Figure 1 , the ANFIS network is composed of five layers. The first layer executes the fuzzification process. The parameters in this layer are referred to as premise parameters. The second layer multiplies the incoming signals from previous layer and sends the product out. Every node in this layer performs the firing strength of fuzzy rules. The third layer normalizes the firing strengths. The fourth layer executes the 
consequent part of the fuzzy rules. Finally, the fifth layer computes the overall output of the fuzzy inference system as the summation of all signals incoming from the fourth layer [25]. The outputs of each layer of the ANFIS are done, separately, by a one of the following equations:

$$
\begin{gathered}
O_{i, 1}^{1}=\mu_{A_{i}}\left(x_{1}\right), \quad i=1,2 \\
O_{i, 2}^{1}=\mu_{B_{i}}\left(x_{2}\right), \quad i=1,2 \\
O_{i}^{2}=w_{i}=\mu_{A_{i}}\left(x_{1}\right) \times \mu_{B_{i}}\left(x_{2}\right) \quad i=1,2 \\
O_{i}^{3}=v_{i}=\frac{w_{i}}{w_{1}+w_{2}}, \quad i=1,2 \\
O_{i}^{4}=v_{i} \times f_{i}=v_{i}\left(a_{i} x_{1}+b_{i} x_{2}+c_{i}\right) \quad i=1,2 \\
O_{i}^{5}=y=\sum_{i=1}^{2} v_{i} \times f_{i}=\frac{\sum_{i=1}^{2} w_{i} \times f_{i}}{\sum_{i=1}^{2} w_{i}}
\end{gathered}
$$

Where $\mu_{A_{i}}\left(x_{1}\right)$ and $\mu_{B_{i}}\left(x_{2}\right)$ are the membership functions. $f_{i}$ are the outputs within the fuzzy region specified by the fuzzy rule, $\left\{a_{i}, b_{i}, c_{i}\right\}$ are the design parameters that are determined during the training process. However, the most commonly used are bell-shaped and gaussian membership functions. For example, if the bell-shaped membership function is employed, $\mu_{\mathrm{A}_{\mathrm{i}}}\left(\mathrm{x}_{1}\right)$ is given by:

$$
\mu_{A_{i}}\left(x_{1}\right)=\frac{1}{1+\left(\left(\frac{x_{1}-c_{i}}{a_{i}}\right)^{2}\right)^{b_{i}}}
$$

\section{PROPOSED ANFIS MODEL}

The adaptive neuro-fuzzy inference system is introduced in this paper to predict the daily load curve of metropolitan France. Hence, collected data from French Transmission System Operator (RTE) website consist of half-hourly recording of the electricity consumption during the period from 01 January 2014 to 27 June 2014. However, to predict the power consumption in a given day L, the proposed model generates first a weekly time series y $(\mathrm{L}-1)$ in which latest forty-eight records represent the electricity demand of the previous day $L-1$. In a similar manner, the model creates seven series that cover the closest weeks to the week $y(L-1)$. Also, three indexes are introduced: time of the week, time of the day and day of the week. Hence, the time series y $(\mathrm{L}-1)$ represents the output of the ANFIS and the others load series and indexes represent the inputs of the ANFIS. However, among ten input vectors, an exhaustive search is performed to extract only two input vectors that most influence on the output. At that stage, selected two inputs are used to generate and trains a Sugeno FIS of four fuzzy rules, two sigmoidal membership function and one hundred epochs. Then, after generating inputs/output vectors associated to the day L, selected two inputs for the day $\mathrm{L}$ are used to perform day-ahead electricity demand forecasting. The flow chart of the proposed method is presented in Figure 2. 


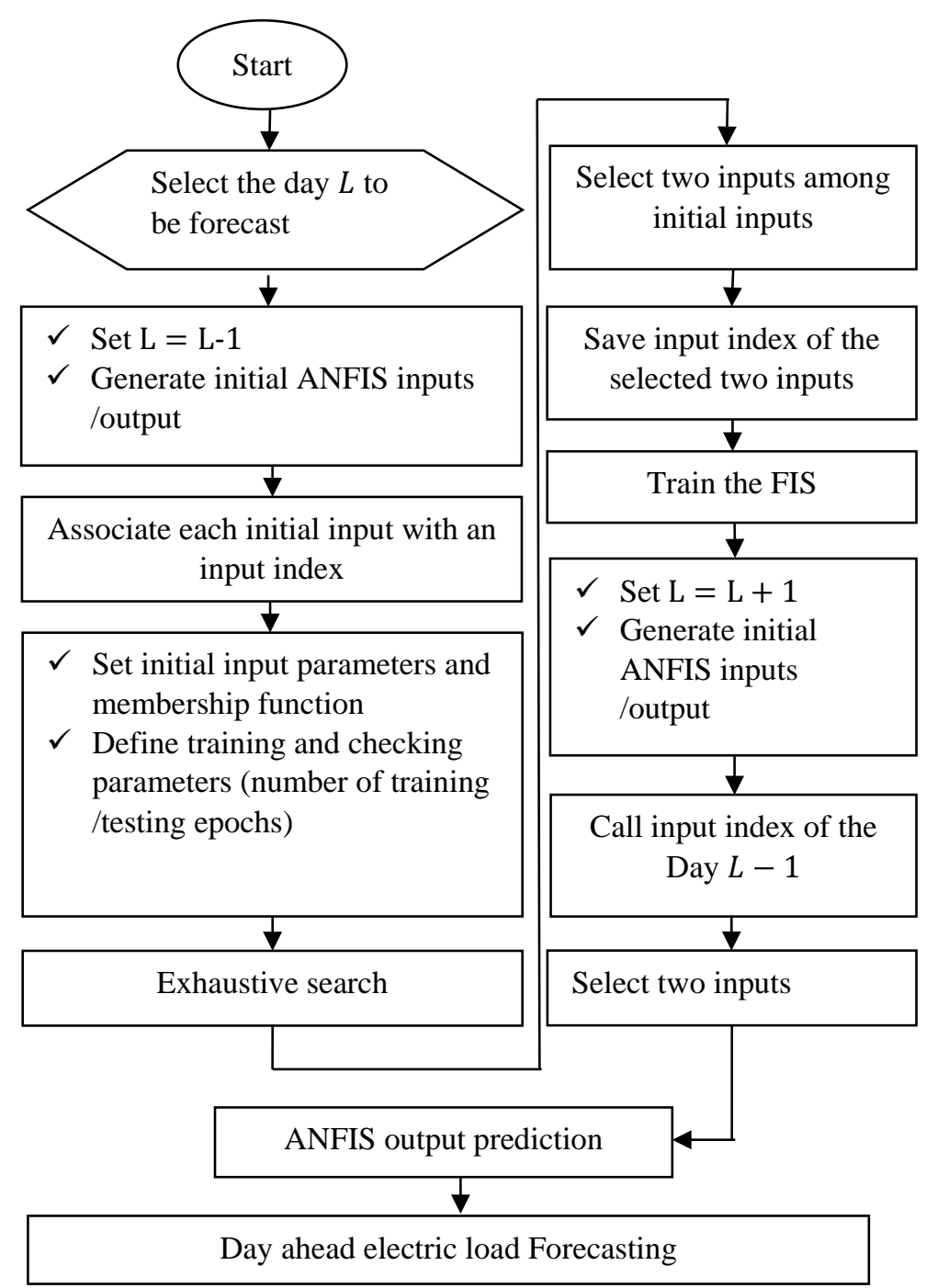

Figure 2. Flow chart of the proposed ANFIS model

\section{RESULTS AND DISCUSSION}

The ANFIS approach discussed in this current study is applied to the electricity demand data of mainland France. These data consist of half-hourly records ranging from Wednesday 01 January 2014 to Friday 27 June 2014. As mentioned in Figure 3, winter season attests a significant increase on the electricity demand, with a maximum peak demand of 82273 MW, reached on 29 January 2014 at 19:00. This increase on electricity consumption is mainly related to the significant effect of temperature in electricity consummation, since in winter heating systems are intensively used, particularly in the evening period around 19:00. While the winter season reaches high peak records, the summer period is characterized by an important decrease on load demand. For illustration, the maximum peak of $55409 \mathrm{MW}$ occurred on 26 June 2014 at 13:00, represents only $67.348 \%$ of the winter peak mentioned above. This decrease in consumption profile is mainly related to decreases in activity due to school holidays during the summer. Nevertheless, the electricity consumption in summer is also affected by meteorological conditions, under the effect of air conditioning and ventilation. However, climatic conditions are ignored in this paper and the proposed algorithm attempts to recognize seasonal effects such as daily and weekly cycles as a basis for the forecast. 


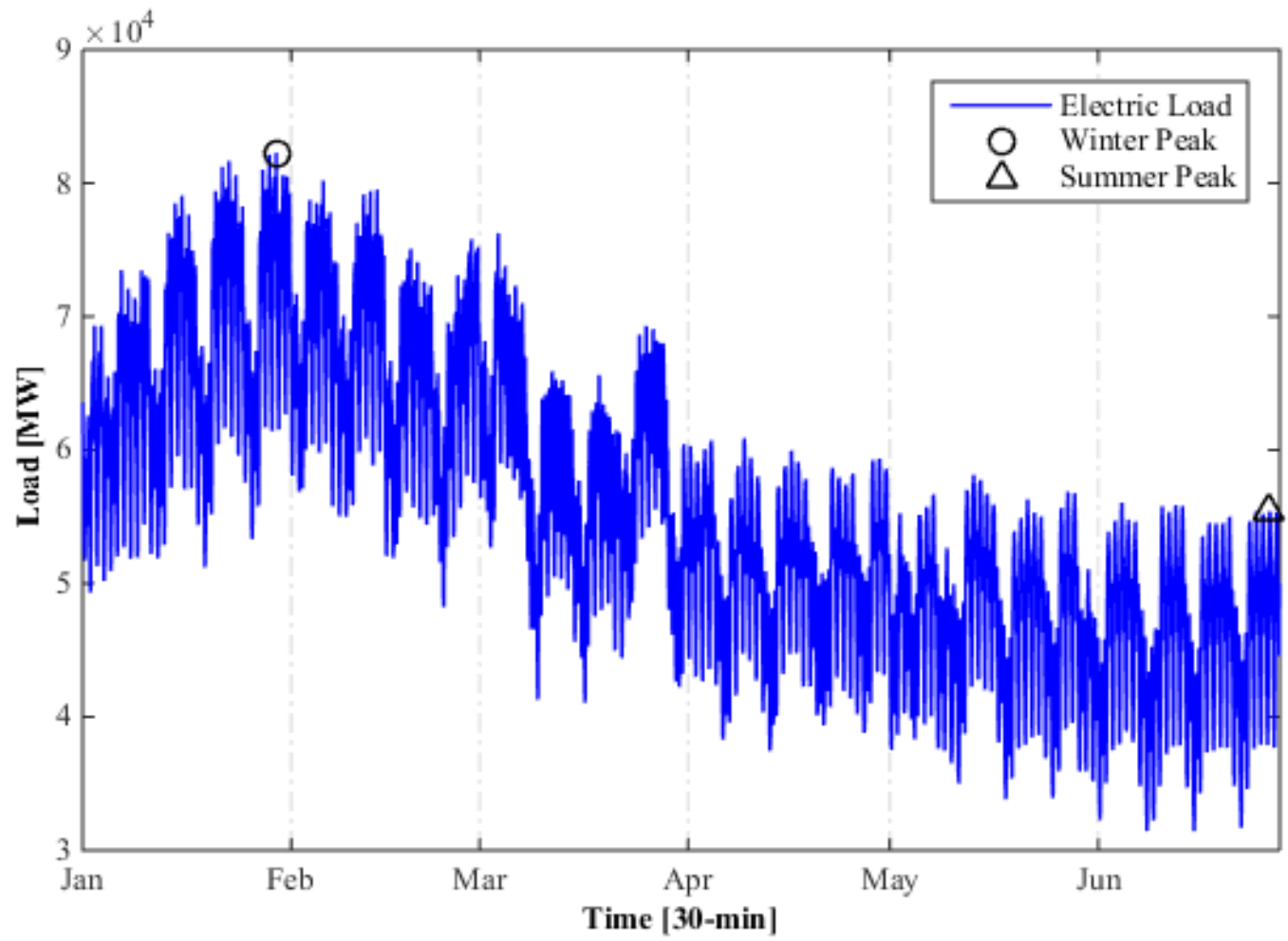

Figure 3. Electric load in France from 01 January 2014 to 27 June 2014

As shown in Figure 4, user-friendly graphical interface is proposed to ensure daily load curve prediction. This graphical interface allows connecting to the transmission system operator website and updating the database, which makes the proposed model in this paper able to be used for online electric load forecasting.

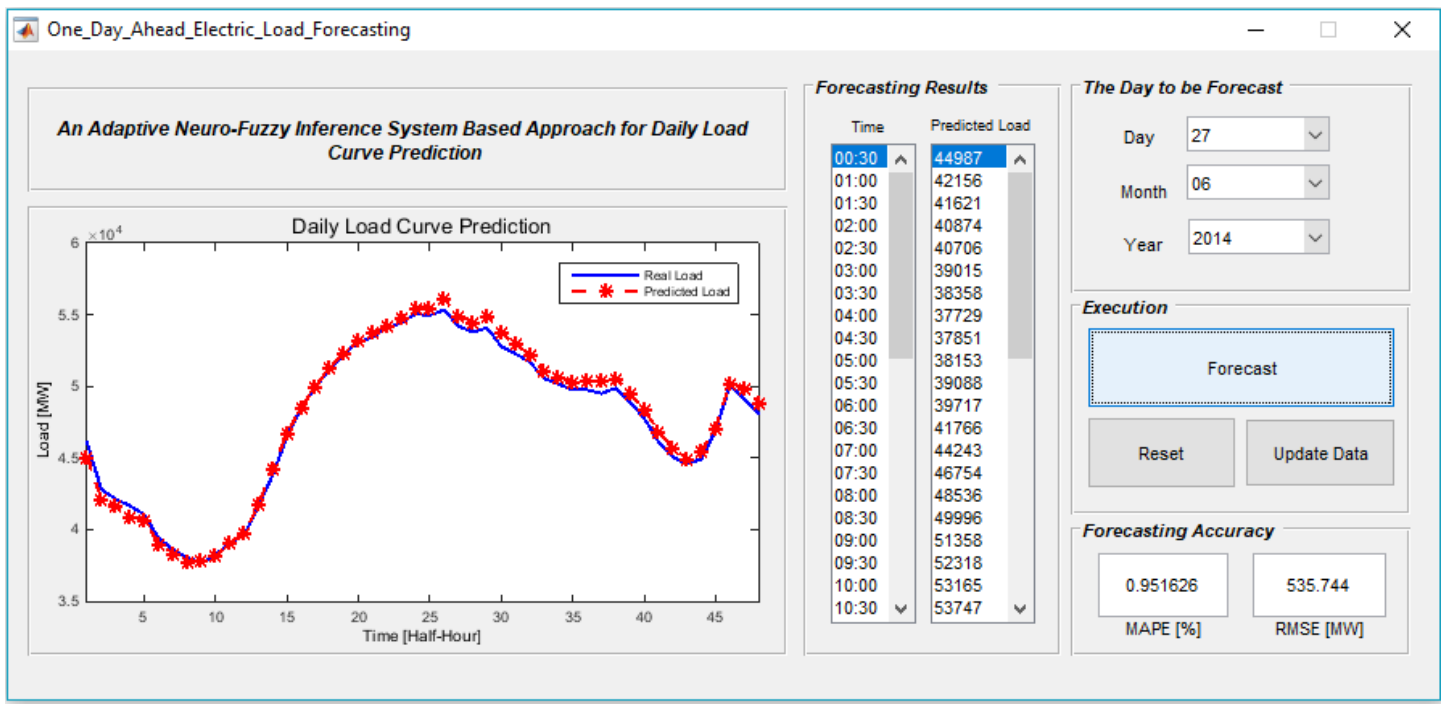

Figure 4. Developed forecasting tool for day ahead electric load forecasting using ANFIS

To evaluate the performance of the proposed electricity demand forecasting system, forecasts are done for day-ahead prediction along two weeks: the first week of spring 2014 (20-26 March 2014) and the first week of summer 2014 (21-27 June 2014). Forecasting results for the two weeks cited above are presented in Figures 5. The upper subplot of Figure 5 shows the results of the proposed adaptive neuro- 
fuzzy inference system-based approach for next-day load curve prediction in France along the week from 20th to 26th March 2014, while lower subplot shows the results of the proposed ANFIS model for daily load curve prediction along the week from 21st to 27th June 2014. For both weeks, the figure shows that satisfactory results were obtained by using the proposed forecasting algorithm, since the shape of predicted daily load curves is very similar to that of real daily load curves and the difference between these curves is almost minimal.
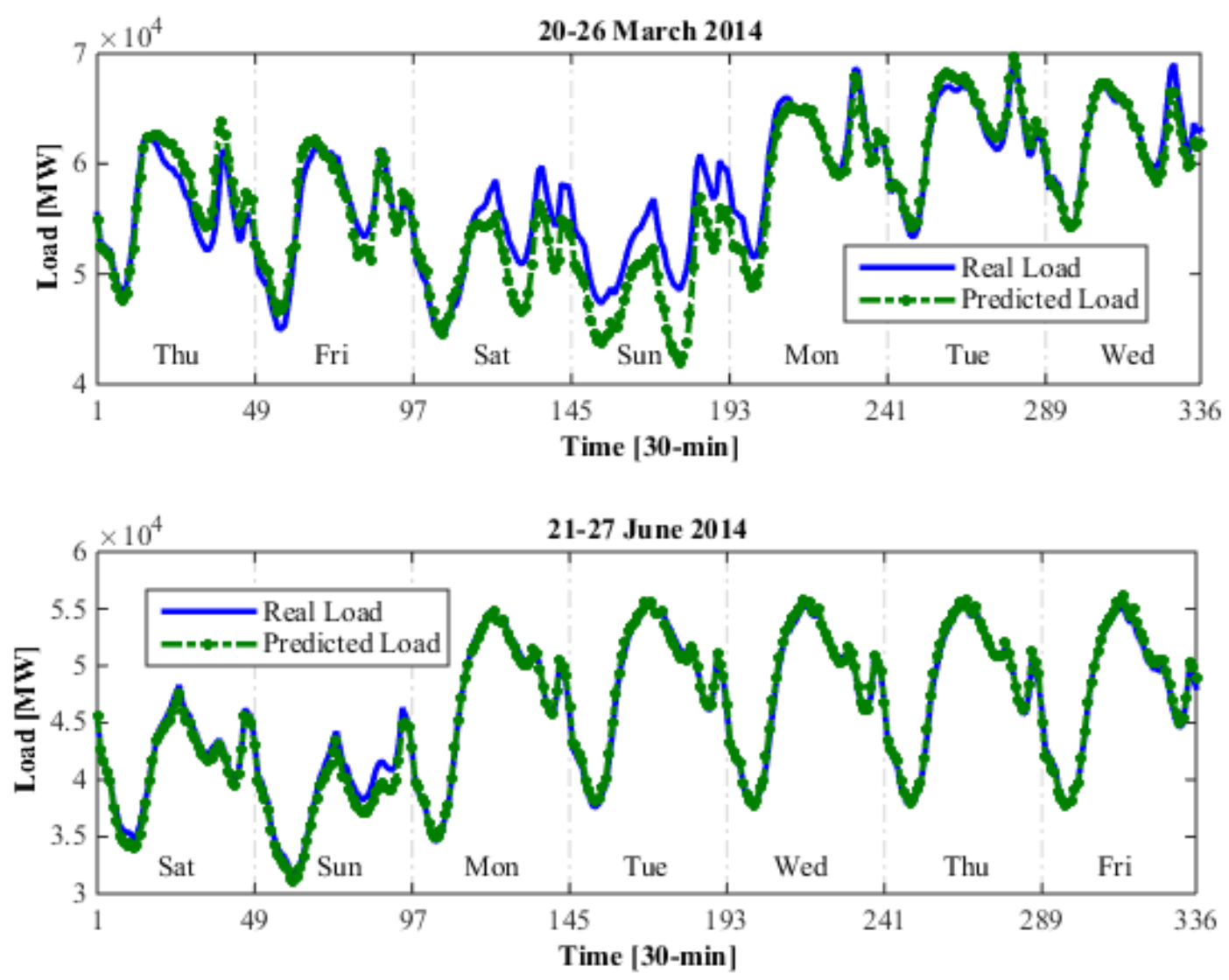

Figure 5. Daily load curve prediction over two weeks

The achieved results in Figure 5 validate the clear benefit of the proposed ANFIS model. This model is based on the principle of inputs selection and daily and weekly cycles modeling. For instance, to predict the daily load curve of 20 March 2014, an exhaustive search such as presented in Figure 6 is performed first to select only two inputs among initial ten inputs. The exhaustive search builds a neuro-fuzzy system, trains it for twenty epochs and reports the overall performance reached. Selected model should confirm the minimum errors while providing outputs predictions. This task of inputs selection plays a key role since the final forecasting accuracy of any algorithm is mainly influenced by the quality of inputs provided to the model [25]. After a primary part for selecting two appropriate input vectors, the second part of our forecasting algorithm is introduced to identify the appropriate architecture of the ANFIS. For the forecasting process of 20 March 2014, Figure 7.a shows that the fuzzy inference system parameters and structure are identified by applying neural learning rules and the optimal topology of the ANFIS is obtained after only nine epochs. Hence, Figure 7.b illustrates the excellent training since the ANFIS output prediction matches well with training and checking data. The final step of the proposed method consists in providing day-ahead electricity demand forecasting. As presented by the upper subplot of Figure 5, one can clearly perceive that the proposed model was able to successfully predict the daily electricity demand of 20 March 2014, since the shape of predicted load curve follows well the shape of actual load curve. 


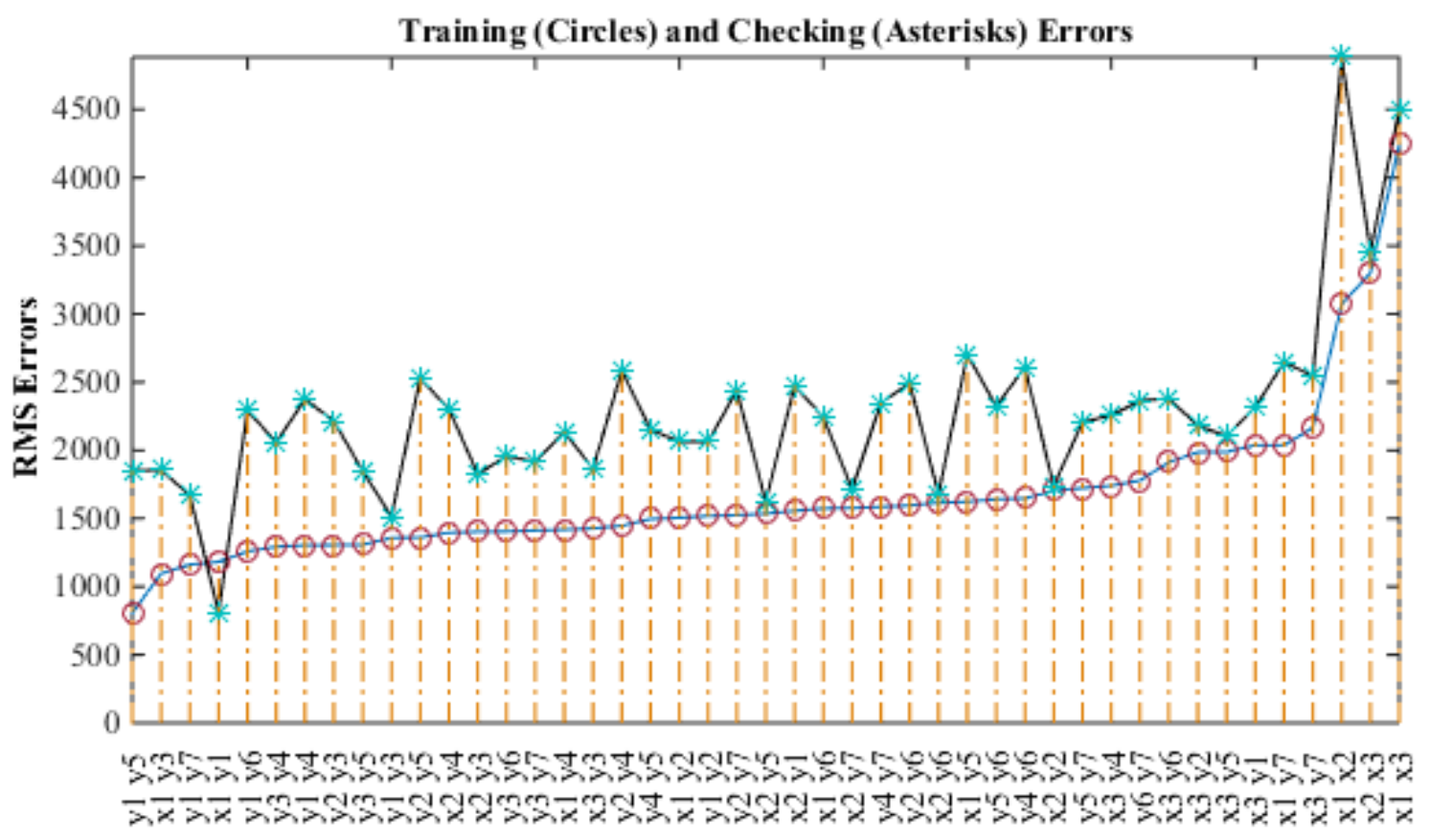

Figure 6. Inputs selection in daily load curve prediction of 20 March 2014
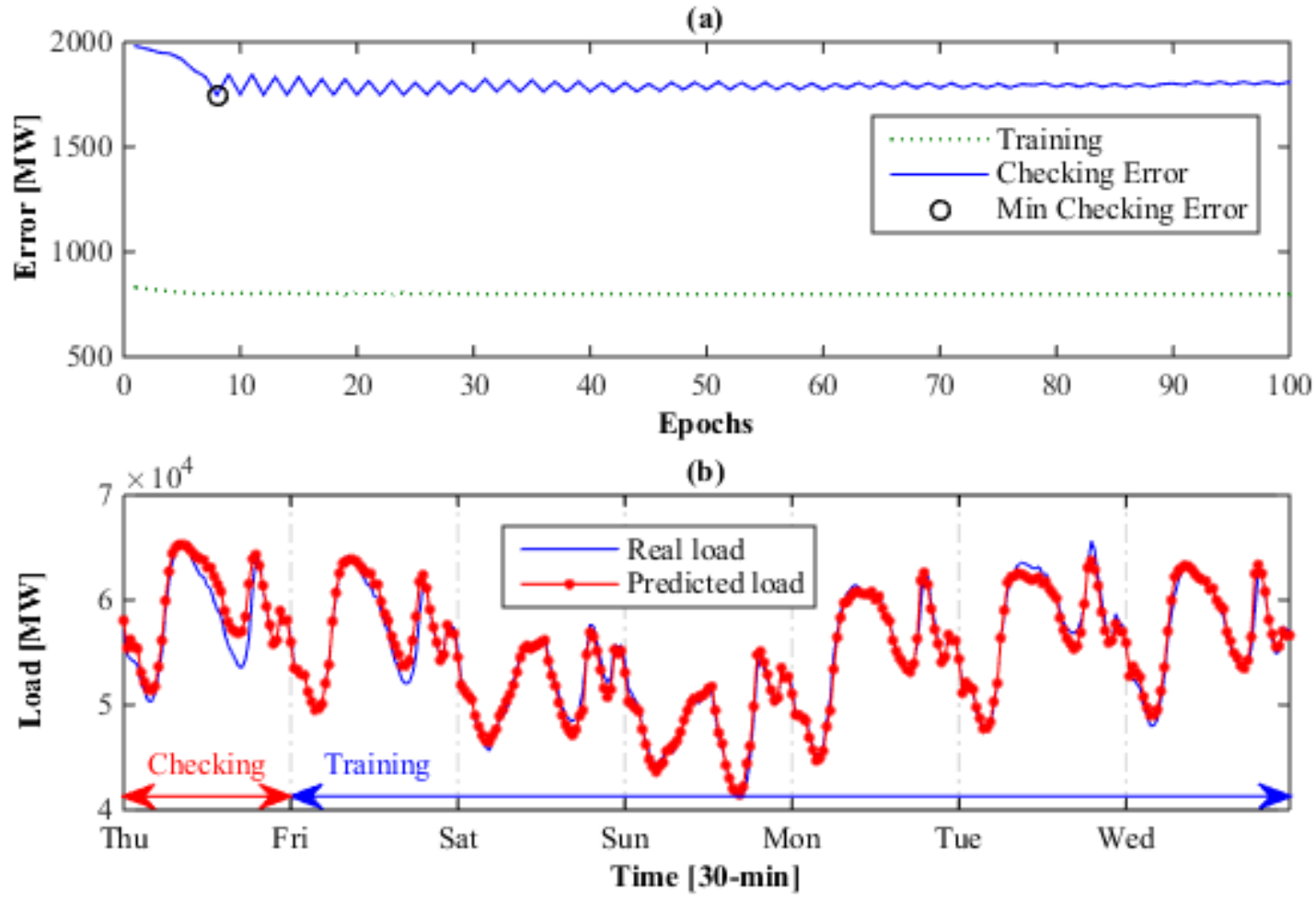

Figure 7. (a) Training and checking errors in the daily load curve prediction of 20 March 2014 (b) ANFIS Training and checking data prediction: 13-19 March 2014 
Table 1. Forecasting accuracy of the proposed ANFIS Model

\begin{tabular}{|c|c|c|c|c|c|c|}
\hline Day\Accuracy & \multicolumn{3}{|c|}{ MAPE [\%] } & \multicolumn{3}{|c|}{ RMSE [MW] } \\
\hline 20 Mar 2014 & 2.853 & & \multirow{14}{*}{2.087} & 1889.80 & & \multirow{14}{*}{1665.45} \\
\hline 21 Mar 2014 & 2.136 & & & 1405.17 & & \\
\hline $22 \operatorname{Mar} 2014$ & 4.131 & & & 2676.13 & & \\
\hline 23 Mar 2014 & 8.023 & 3.082 & & 4374.10 & 2278.18 & \\
\hline $24 \operatorname{Mar} 2014$ & 2.067 & & & 1650.47 & & \\
\hline 25 Mar 2014 & 1.130 & & & 792.095 & & \\
\hline 26 Mar 2014 & 1.236 & & & 1067.20 & & \\
\hline 21 Jun 2014 & 1.350 & & & 617.908 & & \\
\hline 22 Jun 2014 & 2.406 & & & 1066.13 & & \\
\hline 23 Jun 2014 & 0.500 & & & 288.938 & & \\
\hline 24 Jun 2014 & 0.953 & 1.091 & & 502.516 & 597.807 & \\
\hline 25 Jun 2014 & 0.723 & & & 411.081 & & \\
\hline 26 Jun 2014 & 0.755 & & & 437.192 & & \\
\hline 27 Jun 2014 & 0.952 & & & 535.744 & & \\
\hline
\end{tabular}

To measure the forecasting accuracy of the proposed model, we used the Mean Absolute Percentage Error (MAPE) and the Root Mean Squared Error (RMSE) criteria:

$$
\begin{gathered}
\text { MAPE }=\frac{1}{n} \sum_{t=1}^{n} \frac{\left|\widehat{y}_{t}-y_{t}\right|}{y_{t}} \times 100 \\
R M S E=\frac{1}{n} \sqrt{\sum_{t=1}^{n}\left(\widehat{y}_{t}-y_{t}\right)^{2}}
\end{gathered}
$$

Where:

$\hat{y}_{t}:$ Predicted load for time $t$.

$\mathrm{y}_{\mathrm{t}}$ : Actual load at the time $t$.

$n$ : Total number of forecasting cases.

Detailed evaluation results for both weeks are summarized in Table 1. The results in this table show that ANFIS model achieved overall prediction errors as follows: (1) MAPE $=3.082 \%$ and RMSE $=2278.18$ MW for the week from 20th to 26th March 2014, (2) MAPE $=1.091 \%$ and RMSE $=597.807$ MW for the week from 21st to 27th June 2014. For both weeks, one can perceive that the proposed ANFIS model has reached satisfactory performance in the daily load curve prediction process, since the average mean absolute percentage error is equal to $2.087 \%$ and the average root mean squared error is equal to 1665.45 MW. However, to assess the effectiveness of the proposed forecasting scheme, we also propose to compare the performance of ANFIS model against the performance of a set of forecasting methods. This comparison includes the Holt-Winters (HW) exponential smoothing method, Seasonal Autoregressive Integrated Moving Average (SARIMA) model and the well-known Feed Forward Neural Network (FFNN) approach. Exponential smoothing methods and time series approaches are, certainly, the most popular classical methods that have been used in the electricity demand forecasting literature. This reputation is attributed to the ability of these methods to manage the seasonal components of the electric load time series. In the other hand, artificial neural network models are among the artificial intelligence algorithms that have excellent capacity to model nonlinearity and complex relationships that exist 
between the electricity consumption and the other influencing parameters. In this context, it is natural to include the mentioned three forecasting models in our comparison.
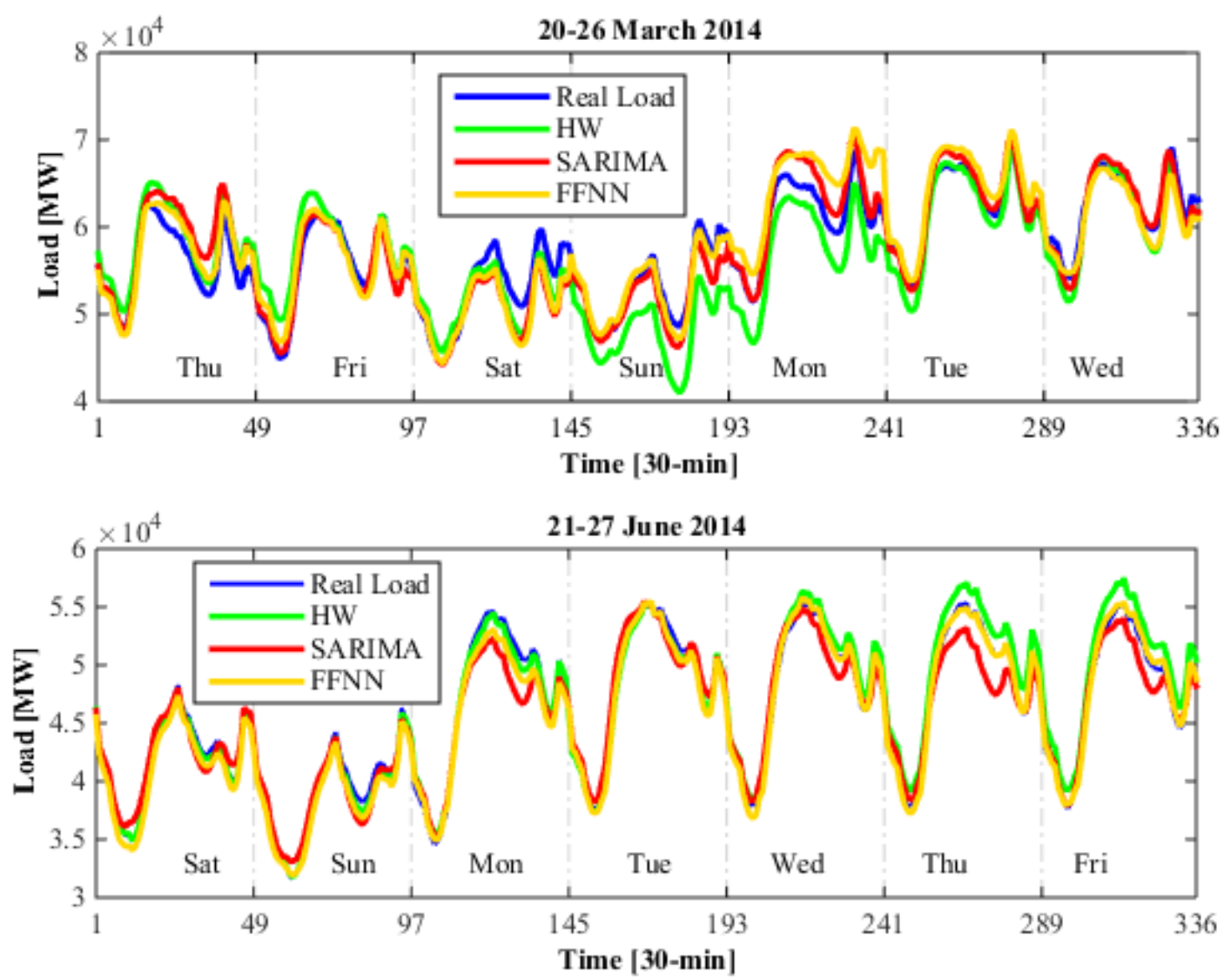

Figure 8. Daily load curve prediction for HW, SARIMA and FFNN

The forecasting results of HW, SARIMA and FFNN are illustrated in Figure 8, while the comparison against ANFIS model is presented in Table 2. It can be seen from the results in Table 2 that the average MAPE of ANFIS is less than all the average MAPEs of the other methods. It can be also noticed that the overall improvement against FFNN, SARIMA and HW is respectively equal to $3.750 \%, 16.784 \%$ and $55.509 \%$. Therefore, the proposed approach is considered to be suitable for daily load curve prediction in practical application.

Table 2. The overall forecasting results of all considered models

\begin{tabular}{lllll}
\hline Methods $\backslash$ Results & HW & SARIMA & FFNN & ANFIS \\
\hline MAPE [\%] & 3.245 & 2.437 & 2.165 & 2.087 \\
RMSE [MW] & 2319.18 & 1709.88 & 1681.91 & 1665.45 \\
\hline
\end{tabular}

\section{CONCLUSION}

We have proposed in this paper an adaptive neuro-fuzzy inference system-based approach for next day electricity demand forecasting. The key idea of the proposed methodology is to attempt by considering seasonal effects such as daily and weekly cycles to capture the main patterns of the electric load time 
series. In order to improve forecasting accuracy, an exhaustive search is performed to select the appropriate inputs that most influence on the output. The obtained results and forecasting performance showed that: (1) the average MAPE of proposed ANFIS model is equal to 2.087\%; (2) ANFIS has better forecasting performance than other methods considered in this study. Therefore, the proposed approach is considered to be suitable for daily load curve prediction in practical application.

\section{REFERENCES}

[1] Parlos AG, Oufi E, Muthusami J, Patton AD, Atiya AF. Development of an intelligent long-term electric load forecasting system. In: ISAP '96. International Conference on Intelligent Systems Applications to Power Systems; 28 Jan-2 Feb 1996: IEEE, Orlando, FL, USA, pp. 288-292.

[2] Parlos AG, Patton AD. Long-term electric load forecasting using a dynamic neural network architecture. In: APT 93. Joint International Power Conference Athens Power Tech; 5-8 Sep 1993: IEEE, Athens, Greece, pp. 816-820.

[3] Dalvand MM, Azami S, Tarimoradi H. Long-term load forecasting of Iranian power grid using fuzzy and artificial neural networks. In: UPEC 2008. 43rd International Universities Power Engineering Conference; 1-4 Sept 2008: IEEE, Padova, Italy, pp. 1-4.

[4] Hong, T, Wilson, J, Xie, J. Long Term Probabilistic Load Forecasting and Normalization with Hourly Information. IEEE Transactions on Smart Grid 2014; 5(1): 456-462 <https://doi.org/10.1109/TSG.2013.2274373>

[5] Jain A, Satish B. Short term load forecasting by clustering technique based on daily average and peak loads. In: PES '09. IEEE General Meeting Power \& Energy Society; 26-30 July 2009: IEEE, Calgary, AB, Canada, pp. 1-7.

[6] Amjady, N, Keynia, F. Mid-term load forecasting of power systems by a new prediction method. Energy $\begin{array}{lllll}\text { Conversion } & \text { and } & \text { Management } & \text { 2008; } & \text { 49(10): }\end{array}$ <https://doi.org/10.1016/j.enconman.2008.04.008>

[7] Jaramillo-Morán, MA, Romera, EG, Fernández, DC. Monthly electric demand forecasting with neural filters. International Journal of Electrical Power \& Energy Systems 2013; 49: 253-263 <https://doi.org/10.1016/j.ijepes.2013.01.019>

[8] Torkzadeh R, Mirzaei A, Mirjalili MM, Anaraki AS, Sehhati MR, Behdad F. Medium term load forecasting in distribution systems based on multi linear regression \& principal component analysis: A novel approach. In: EPDC 2014. 19th Conference on Electrical Power Distribution Networks; 6-7 May 2014: IEEE, Tehran, Iran, pp. 66-70.

[9] Laouafi, A, Mordjaoui, M, Haddad, S, Boukelia, TE, Ganouche, A. Online electricity demand forecasting based on an effective forecast combination methodology. Electric Power Systems Research 2017; 148: 3547 <https://doi.org/10.1016/j.epsr.2017.03.016>

[10] Charytoniuk, W, Chen, MS. Very Short-Term Load Forecasting Using Artificial Neural Networks. IEEE Transactions on Power Systems 2000; 15: 263-268 <https://doi.org/10.1109/59.852131>

[11] Hesham, K. Electric load forecasting: Literature survey and classification of methods. International Journal of Systems Science 2012; 33(1): 23-34 <https://doi.org/10.1080/00207720110067421>

[12] Box GEP, Jenkins JM. Time Series Analysis: Forecasting and Control. In: Holden-Day, San Francisco, 1976.

[13] Hagan, MT, Behr, SM. The Time Series Approach to Short Term Load Forecasting. IEEE Transactions on Power Systems 1987; 2(3): 785-791 <https://doi.org/10.1109/TPWRS.1987.4335210>

[14] Papalexopoulos, AD, Hesterberg, TC. A regression-based approach to short-term system load forecasting. IEEE Transactions on Power Systems 1990; 5: 1535-1547 <https://doi.org/10.1109/PICA.1989.39025>

[15] Taylor, JW. Short-Term Load Forecasting with Exponentially Weighted Methods. IEEE Transactions on Power Systems 2012; 27(1): 458-464 <https://doi.org/10.1109/TPWRS.2011.2161780>

[16] Park, DC, El-Sharkawi, MA, Marks, RJII, Atlas, LE, Damborg, MJ. Electric load forecasting using an artificial neural network. IEEE Transactions on Power Systems 1991; 6(2): 442-449 <https://doi.org/10.1109/59.76685>

[17] Ranaweera, DK, Hubele, NF, Karady, GG. Fuzzy logic for short term load forecasting. International Journal of Electrical Power \& Energy Systems 1996; 18(4): 215-222 <https://doi.org/10.1016/01420615(95)00060-7> 
[18] Zhang, X, Wang, J. A novel decomposition-ensemble model for forecasting short-term load-time series with multiple seasonal patterns. Applied Soft Computing 2018; 65: 478-494 <https://doi.org/10.1016/j.asoc.2018.01.017>

[19] Mordjaoui, M, Haddad, S, Medoued, A, Laouafi, A. Electric load forecasting by using dynamic neural network. International Journal of Hydrogen Energy 2017; 42(28): 17655-17663 <https://doi.org/10.1016/j.ijhydene.2017.03.101>

[20] Palit AK, Anheier W, Popovic D. Electrical Load Forecasting Using a Neural-Fuzzy Approach. In: Chiong R, Dhakal S, editors. Natural Intelligence for Scheduling, Planning and Packing Problems, Springer-Verlag, Berlin Heidelberg, 2009. pp. 145-173.

[21] Laouafi, A, Mordjaoui, M, Laouafi, F, Boukelia, TE. Daily peak electricity demand forecasting based on an adaptive hybrid two-stage methodology. International Journal of Electrical Power \& Energy Systems 2016; 77: 136-144 <https://doi.org/10.1016/j.ijepes.2015.11.046>

[22] Palit AK, Popovic D. Computational Intelligence in Time Series Forecasting: Theory and Engineering Applications. Springer-Verlag Berlin, Heidelberg, 2005.

[23] Sumathi S, Surekha P. Computational intelligence paradigms theory and applications using MATLAB. Taylor and Francis Group, LLC, 2010.

[24] Jang, JSR. ANFIS: Adaptive network based fuzzy inference system. IEEE Transactions on systems, Man, and, Cybernetics 1993. 23(3): 665-685 <https://doi.org/10.1109/21.256541>

[25] Laouafi A, Mordjaoui M, Dib D. One-Hour Ahead Electric Load Forecasting Using Neuro-fuzzy System in a Parallel Approach. In: Azar AT, Vaidyanathan S, editors. Computational Intelligence Applications in Modeling and Control, Cham, Switzerland: Springer International Publishing, 2015. pp. 95-121. 